\title{
LA RESPONSABILIDAD DEL OFTALMÓLOGO AL EMPLEAR MEDICAMENTOS NO RENTABLES A LOS LABORATORIOS
}

\section{THE RESPONSABILITY OF THE OPHTHALMOLOGIST WHEN USING DRUGS WHICH ARE UNECONOMIC FROM THE PERSPECTIVE OF THE PHARMACEUTICAL INDUSTRY}

\author{
MENÉNDEZ DE LUCAS JA ${ }^{1}$, MORCILLO LAIZ R²
}

Tenemos asumido que el estilo de vida actual implica estar sometido a muchos condicionantes de tipo económico, pero cuando éstos afectan al derecho del paciente a acceder a un medicamento de eficacia demostrada para tratar su enfermedad o al derecho del oftalmólogo a ejercer su profesión en un clima de cierta tranquilidad, creemos que merecen ser motivo de reflexión.

Actualmente se están empleando en oftalmología una serie de medicamentos, que a pesar de existir claras evidencias científicas de su eficacia para tratar determinadas patologías, no están disponibles en forma de especialidades farmacéuticas autorizadas para uso intraocular. El motivo de esta falta de disponibilidad parece ser la ausencia de interés por parte de los laboratorios farmacéuticos en comercializarlos por su escasa rentabilidad o en algunos casos porque podrían competir con algunos productos que les resultan más rentables. Las consecuencias de esta paradójica e injusta situación las están sufriendo tanto los pacientes que tienen limitado su acceso a un recurso terapéutico eficaz y barato para su enfermedad, como los oftalmólogos que se ven obligados a realizar una serie de gestiones administrativas (solicitudes de autorización por vía compasiva, firma de documentos de consentimiento informado adicionales, informe oftalmológico de cada paciente, etc.) e incluso en ocasiones manipulaciones de los fármacos con el consiguiente riesgo de toxicidad que conlleva la farmacia casera o «kitchen pharmacy» de la literatura anglosajona (1), que podrían evitarse si estuvieran disponibles presentaciones comercializadas para uso intraocular.
Comentábamos recientemente los problemas que están teniendo los retinólogos con el empleo de los modernos medicamentos anti-angiogénicos y con la administración intravítrea del acetónido de triancinolona (2). Existe una abrumadora diferencia de precio entre el bevacizumab (Avastin ${ }^{\circledR}$ ) y otros antiangiogénicos: el pegaptanib (Macugen ${ }^{\circledR}$ ) o el ranibizumab (Lucentis ${ }^{\circledR}$ ) (3). Algunos oftalmólogos, para poder proporcionar el bevacizumab (Avastin ${ }^{\circledR}$ ) a pacientes, que por razones de tipo económico no tendrían acceso a las otras terapias antiangiogénicas, se podrían ver obligados a realizar una serie de diluciones, decantaciones y manipulaciones químicas imprescindibles para poder aplicar intraocularmente la preparación que está destinada al uso sistémico. Las consideraciones de tipo económico son muy importantes pero, evidentemente, los criterios médicos y médico-legales deben anteponerse. Si el paciente acabara sufriendo secuelas tras la aplicación de un medicamento no autorizado, la situación sería difícilmente defendible.

La legislación española contempla solamente cuatro formas de administrar un medicamento (1), que son:

1. Medicamento autorizado: son las especialidades farmacéuticas registradas.

2. Medicamento en fase de investigación clínica: en los casos en los que son calificados como tal por la Administración, y limitado al marco de un ensayo clínico concreto y autorizado.

3. Medicamento extranjero: son los medicamentos legalmente comercializados en otros países,

\footnotetext{
1 Doctor por la UCM. Oftalmólogo de la Clínica Médico Forense de Madrid. Fundación Instituto de Ciencias Visuales.

2 Doctor por la UAM. FEA del Hospital Ramón y Cajal (Madrid). Instituto de Ciencias Visuales (Madrid).

E-mail: incivi@incivi.com
} 
imprescindibles para ese caso y autorizados por el Ministerio.

4. Medicamento de uso compasivo cuando lo indique un especialista hospitalario, a un paciente en el que no existan otras alternativas terapéuticas o ya se hayan agotado y se considere indispensable su utilización.

En el caso del bevacizumab (Avastin ${ }^{\circledR}$ ), no podríamos emplear la vía del uso compasivo. El motivo es que no se cumplen los requisitos que exige la ley que lo regula (4). Así en el artículo 28 del RD 223/2006 se dice textualmente «...cuando el médico, bajo su exclusiva responsabilidad considera indispensable su utilización». No podemos considerar «indispensable» la utilización de un medicamento, mientras existan alternativas terapéuticas disponibles que no hayamos agotado. Actualmente existen al menos dos alternativas comercializadas al bevacizumad, que serían: el pegaptanib (Macugen ${ }^{\circledR}$ ), actualmente disponible en España como especialidad farmacéutica registrada o el ranibizumab (Lucentis ${ }^{\circledR}$ ), recientemente aprobado por la Agencia Europea del Medicamento, por lo que en breve estará también disponible como presentación comercializada en nuestro País.

Una situación parecida ocurre con la aplicación de acetónido de triancinolona (Trigon ${ }^{\circledR}$ Depot) por vía intravítrea, para tratar el edema macular diabético persistente y otros trastornos inflamatorios intraoculares o con proliferación neovascular subretiniana. Este uso obliga al oftalmólogo a realizar una serie de manipulaciones de tipo químico (decantaciones, filtrados,...) de la presentación comercializada. El empleo de la triancinolona intravítrea es habitual y está avalado por multitud de referencias bibliográficas (5) y ensayos clínicos (68). Sin embargo, al no disponer de una presentación comercializada para uso intraocular, debe emplearse mediante la vía del uso compasivo (aquí sí que es indispensable su uso) o como medicamento extranjero (tenemos referencia de dos presentaciones comercializadas para uso intraocular: Aurocort ${ }^{\circledR}$ del laboratorio hindú Aurolab y Visagen ${ }^{\circledR}$ del laboratorio australiano Regenera). El empleo de medicamentos a través de la vía del uso compasivo implica cumplimentar una amplia documentación (informe del oftalmólogo en el que se recogen las terapias previas, la ausencia de alternativas terapéuticas, conformidad del director del hospital, documento de CI y autorización de la Agencia del Medicamento). Pueden solicitarlo especialistas a través del servicio de farmacia hospitalario, para pacientes ingresados o ambulantes. Si no se cumplieran estos trámites y se produjera una complicación, el oftalmólogo podría llegar a encontrarse en una situación comprometida.

Últimamente, la cirugía de polo anterior, también está sufriendo una situación parecida con la aplicación de cefuroxima en la profilaxis de infecciones en cirugía intraocular. Los resultados publicados recientemente por la ESCRS (Sociedad Europea de Cirugía de Cataratas y Refractiva), demuestran que el riesgo de endoftalmitis tras facoemulsificación se reduce casi cinco veces con el empleo de cefuroxima en cámara anterior al final de la cirugía (9). Ante una evidencia científica tan clara frente a un proceso tan grave, lógicamente habrá que modificar los protocolos quirúrgicos incluyendo la aplicación de cefuroxima y probablemente excluyendo otro tipo de medidas profilácticas de eficacia no demostrada (10). Por el momento no hay ningún laboratorio que fabrique una presentación de cefuroxima para uso en cámara anterior, por lo que se está administrando a través de la vía del uso compasivo de medicamentos, que acabamos de describir. El motivo de que no dispongamos de una presentación comercializada, es que al ser un fármaco genérico y barato, los laboratorios no están interesados en realizar los complejos trámites necesarios para lograr la autorización para su uso intraocular (1). Esta situación le supone al cirujano de cataratas una serie de dificultades: tener que cumplimentar la documentación que exige esta vía, hacerle firmar al paciente dos documentos de consentimiento informado (uno para la intervención y otro para la aplicación de cefuroxima), tener que hacer las gestiones necesarias con la farmacia del hospital, etc.

En los tres casos que acabamos de describir, la situación es básicamente la misma: existe un medicamento de eficacia claramente demostrada y admitida por la comunidad científica, que lleva años utilizándose por vía sistémica, pero no está autorizada su aplicación intraocular y no es rentable comercializar este tipo de presentación.

Entendemos que es lícito que los laboratorios farmacéuticos se rijan por criterios de tipo económico, pero en situaciones como las descritas, acaban sufriendo su falta de interés comercial el oftalmólogo y los pacientes. El oftalmólogo, porque ante todo es médico, y no puede permanecer impasible teniendo a su alcance un remedio eficaz para la 
dolencia de su enfermo; pero probablemente los más perjudicados sean los pacientes. Afortunadamente, la mayoría de ellos desconoce que el motivo de que no tengan acceso a un remedio eficaz para su problema es que no les resulta rentable fabricarlo a las multinacionales farmacéuticas.

La solución a este problema, no creemos que sea sencilla. Prueba de ello, es que esta situación también la están sufriendo los colegas de los países de nuestro entorno. Es posible que una forma de remediar esta situación fuera que los oftalmólogos corporativamente, es decir, a través de las sociedades profesionales (SEO, SECOIR,...), solicitáramos al organismo competente de la Administración, la autorización para aplicar estas sustancias concretas por vía intraocular, y su preparación con las debidas garantías sanitarias a través de los Servicios de Farmacia Hospitalarios, como se hace con los colirios reforzados, por ejemplo. Sería una vía para medicamentos concretos de eficacia comprobada en determinadas indicaciones terapéuticas y cuya fabricación no resultara rentable a los laboratorios farmacéuticos, que evitaría los amplios y repetitivos trámites de la vía del uso compasivo y las manipulaciones del oftalmólogo.

Posiblemente a las multinacionales del sector no vean con buenos ojos que se abra una nueva vía para dispensación de medicamentos, fuera de su control y moverán sus poderosas influencias para bloquearla o mantenerla controlada. De cualquier modo, no podemos dejar de denunciar la situación y proponer alguna solución que no implique obligar al oftalmólogo a asumir un riesgo innecesario, desde el punto de vista médico legal, o restringir al paciente un recurso terapéutico de eficacia comprobada para su enfermedad, porque no resulta rentable su fabricación.

\section{BIBLIOGRAFÍA}

1. Abreu JA, Alió JL, Cordovés LM, Ferrer C. Estudio multicentrico europeo para la prevención de la endoftalmitis en la cirugía de catarata. Arch Soc Esp Oftalmol 2006; 81: 627-630.

2. Menéndez de Lucas JA, Morcillo Laiz R. Cuestiones médico-legales sobre los fármacos actualmente empleados en el tratamiento de la DMAE. Arch Soc Esp Oftalmol 2006; 81: 359-362.

3. Michels S, Rosenfeld PJ, Puliafito CA, Marcus EN, Venkatraman AS. Systemic bevacizumab (Avastin) therapy for neovascular age-related macular degeneration twelveweeks results of an uncontrolled open-label clinical study. Ophthalmology 2005; 112: 1035-1047.

4. Real Decreto 223/2004, de 6 de febrero, por el que se regulan los ensayos clínicos con medicamentos. BOE $n .^{o}$ 33 de 27 de febrero de 2004; 325.

5. Domínguez A, Gómez-Ulla FJ, Ruiz JM, Benítez JM, Agencia E. Antiinflamatorios y antiangiogénicos intraoculares. Mesa redonda 80 congreso de la Sociedad Española de Oftalmología. Madrid: MacLine SL; 2004.

6. Gillies MC, Simpson JM, Luo W, Penfold P, Hunyor AB, Chua $W$, et al. A randomized clinical trial of a single dose of triamcinolone acetonide for neovascular age-related macular degeneration: one-year results. Arch Opthalmol 2003; 121: 667-673.

7. Jonas JB, Akkoyun I, Budde WM, Keissig I, Degenring $R F$. Intravitreal re-injection of triamcinolone for exudative age-related macular degeneration. Arch Ophthalmol 2004; 122: 218-222.

8. Jonas JB. Intravitreal triamcinolone acetonide for treatment of intraocular oedematous and neovascular diseases. Acta Ophthalmol Scand 2005; 83: 645-663.

9. Barry P, Seal DV, Gettinby $G$, Lees $F$, Peterson $M$, Revie CW. ESCRS study of prophylaxis of postoperative endophthalmitis in cataract surgery: Preliminary report of principal results from a European multicenter study. $J$ Cataract Refract Surg 2006; 32: 407-410.

10. Menéndez JA. Aspectos médico legales de las endoftalmitis. En: Arias A. Profilaxis y tratamiento de la infección quirúrgica en oftalmología. Mesa redonda 82 congreso de la Sociedad Española de Oftalmología. Madrid: MacLine SL; 2006; 152-166. 